Baltic Astronomy, vol. 10, 651-669, 2001.

\title{
THE DEPENDENCE OF ATMOSPHERIC EXTINCTION ON METEOROLOGICAL CONDITIONS AND AEROSOL SIZE DISTRIBUTION
}

\author{
Erika Pakštienè \\ Institute of Theoretical Physics and Astronomy, \\ Goštauto 12, Vilnius 2600, Lithuania
}

Received August 24, 2001.

\begin{abstract}
Based on seven years of photometric observations collected at Moletai Observatory, the dependence of atmospheric extinction on the meteorological conditions (pressure, temperature, absolute and relative humidity) is discussed. Using the observed extinction coefficients and Mie theory of light scattering, the Junge size distribution of the aerosol particles were evaluated for Moletai (Lithuania) and Maidanak (Uzbekistan) observatories. They were used to determine the dependence of atmospheric extinction on aerosol concentration and Junge coefficients $\mathrm{u}$ and $\mathrm{v}$ and the aerosol extinction parameters $A_{0}$ and $b$ were found. The dependences $v(b)$ and $u\left(A_{0}, b\right)$ can be used to derive a rough estimate of the Junge size distribution for every moment of extinction observation.
\end{abstract}

Key words: atmospheric extinction - methods: observational techniques: photometric (Vilnius photometric system)

\section{INTRODUCTION}

Extinction in the Earth's atmosphere raises many problems in accurate ground-based stellar photometry. It depends on many factors, including the geographical location of observing site. Atmospheric extinction is closely connected with the latitudinal dependence of the thickness of ozone layer (McCartney 1977, Zujev \& Komarov 1986), the dependence of Rayleigh scattering on the altitude above sea level (Hayes \& Latham 1975), and site conditions (Lockwood \& Thompson 1986) relating to a specific structure 
and size distribution of the aerosol particles (Gutiérrez-Moreno et al. 1982, Reimann et al. 1992). For instance, the atmospheric extinction at Teides Observatory (Tenerife, Canary Islands) is sometimes augmented by dust clouds blown from the Sahara desert (Jiménez et al. 1998). In such condition the second maximum appears in aerosol size distribution, corresponding to particle sizes of about $0.7 \mu \mathrm{m}$. At Moletai Observatory, Lithuania, the unstability of atmospheric extinction is mainly due to the Atlantic influence and, also, to low altitude $(0.2 \mathrm{~km}$ above sea level $)$ of the site. There are usually small water droplets or other small particles of different nature (pollen, smoke, etc.) in the atmosphere above Moletai. Atmospheric extinction conditions at the high-altitude Maidanak Observatory (2.5 $\mathrm{km}$ above sea level), Uzbekistan, differ significantly from those at Moletai. Rayleigh extinction at Maidanak is lower and the nature of aerosols appears to be different. Due to very low relative humidity (usually less than 50 percent), regular aerosols at this high-altitude site are mostly solid particles. As in the case of Teide Observatory, most of the extinction growth is produced by silicate dust transported by a southwest wind from the Afghanistan deserts.

In this paper, which is the third contribution within a series of four papers (henceforth, called Papers I to IV), the dependence of atmospheric extinction on meteorological conditions (pressure, temperature, absolute and relative humidity) at Molètai Observatory is discussed (Section 3). Also, using the extinction coefficients described in Paper II (Pakštiene et al. 2001) and Mie theory of light scattering (Dave 1969, Yamamoto \& Tanaka 1969, Wiscombe 1980, Heintzenberg et al. 1981, de Rooij \& van der Stap 1984, Shah 1985, Trakhovsky \& Shettle 1987, Forbes et al. 1996, Jiménez et al. 1998) Junge size distributions of the aerosol particles are evaluated for Molètai and Maidanak Observatories. And, finally, by applying these distributions, the dependence of the atmospheric extinction on aerosol concentration and Junge coefficients is determined (Section 4).

\section{OBSERVATIONS AND INITIAL DATA}

The extinction coefficients, used for this investigation, were determined from photometric observations in the Vilnius system, carried out at Moletai in 1992-1999 and at Maidanak in 1978-1998. Details of the observations used are given in Paper II. 
Meteorological data (temperature, atmospheric pressure and humidity), given for every 3 hours, were taken from the Utena meteorological station situated about $23 \mathrm{~km}$ north of Molètai Observatory. These were interpolated for the moments of extinction observations. We suppose that the meteorological conditions at Moletai Observatory are practically the same as in Utena. The distributions of temperature, pressure, absolute and relative and humidity are shown in Fig. 1.
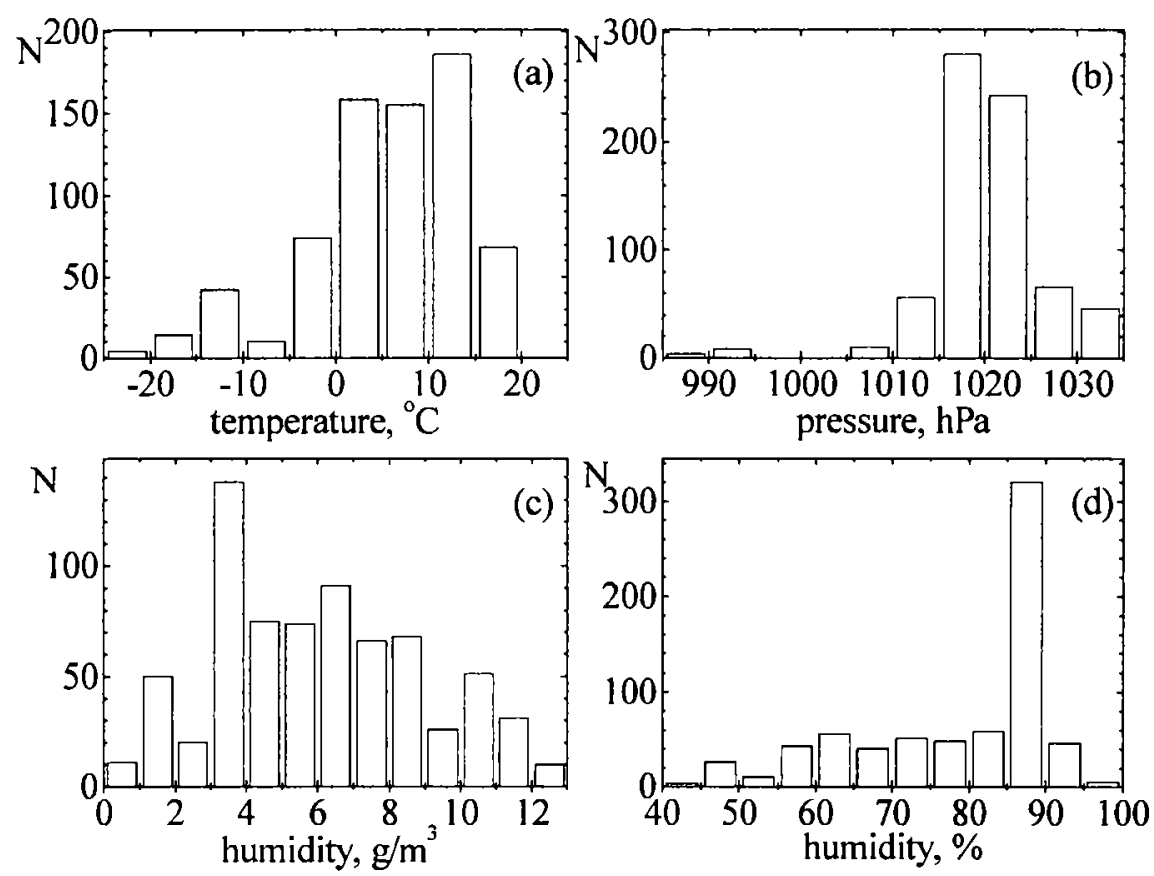

Fig. 1. Distributions of meteorological parameters at Molètai Observatory during the time of extinction observations.

\section{THE DEPENDENCE OF EXTINCTION ON METEOROLOGICAL CONDITIONS AT MOLETAI}

The dependence of the mean extinction coefficients for three color indices on meteorological parameters (temperature, pressure, absolute and relative humidity) is shown in Fig. 2. The intervals into which the values of parameters were binned and for which the mean extinction coefficients $\alpha$ were calculated, are given in the figure 
caption. The vertical bars indicate the standard deviation from the mean value.

\subsection{Dependence on temperature}

As we can see from Figure 2, the dependence of all extinction coefficients on both the temperature (Fig. 2 a) and absolute humidity (Fig. $2 \mathrm{c}$ ) is much the same: when the temperature, or absolute humidity, is rising, the extinction coefficients increase. This may be due to the fact that, with rising temperature, the density of saturated vapor becomes larger. So, the dependence of extinction on temperature and absolute humidity may, in fact, be conditioned by changes in only one parameter, temperature or absolute humidity. To check which of the two parameters, temperature or absolute humidity, plays the main role in extinction variations, an additional test was made. The extinction coefficients were divided into groups of narrow absolute humidity intervals, and then a temperature dependence within each group was examined. Two of the cases, when humidity was 1 to $2 \mathrm{~g} / \mathrm{m}^{3}$, and 6 to $7 \mathrm{~g} / \mathrm{m}^{3}$, are shown in Fig. 3 (a and $\mathrm{b}$, respectively). The most important features to note in the figure are that at low temperature and low absolute humidity, practically no temperature dependence is seen (Fig. 3a), but in the case when both the temperature and absolute humidity are high, the temperature dependence clearly exists (Fig. 3b). The extinction coefficient $\alpha_{U-V}$ increases by about $0.1 \mathrm{mag}$ when the temperature rises by $14^{\circ} \mathrm{C}$. At lower absolute humidity and temperature, the dependence of extinction on temperature is also perceptible. Therefore we can conclude that the atmospheric extinction increases with rising temperature, and that this increase becomes large at higher temperatures. The same tendency is seen in Fig. 2a, where the mean extinction values are given plotted for every five-degree interval.

\subsection{Dependence on humidity}

It is known that optical properties of aerosols, such as light scattering by these particles, depend on the relative humidity of air. $\mathrm{Hu}-$ midity has an effect upon light scattering by aerosols when relative humidity gets higher than 20-30 percent. However, this effect is not the same for different climatic conditions and seasons. In addition, the relation between aerosol scattering properties and relative hu- 

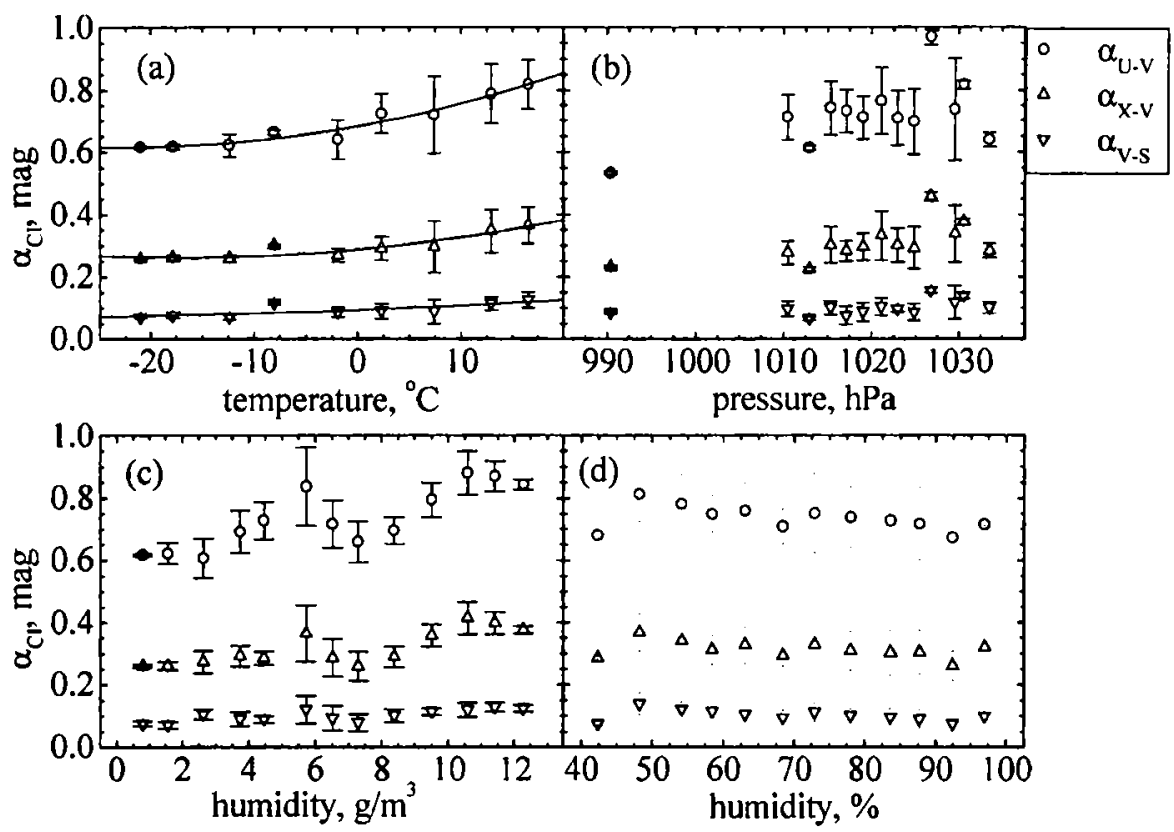

Fig. 2. Dependence of the mean extinction coefficients for three color indices on meteorological parameters: (a) temperature (means calculated for $5^{\circ} \mathrm{C}$ intervals), (b) pressure (means for $2 \mathrm{hPa}$ intervals), (c) absolute humidity (means for $2 \mathrm{~g} / \mathrm{m}^{3}$ intervals), and (d) relative humidity (means for 5 per cent intervals).

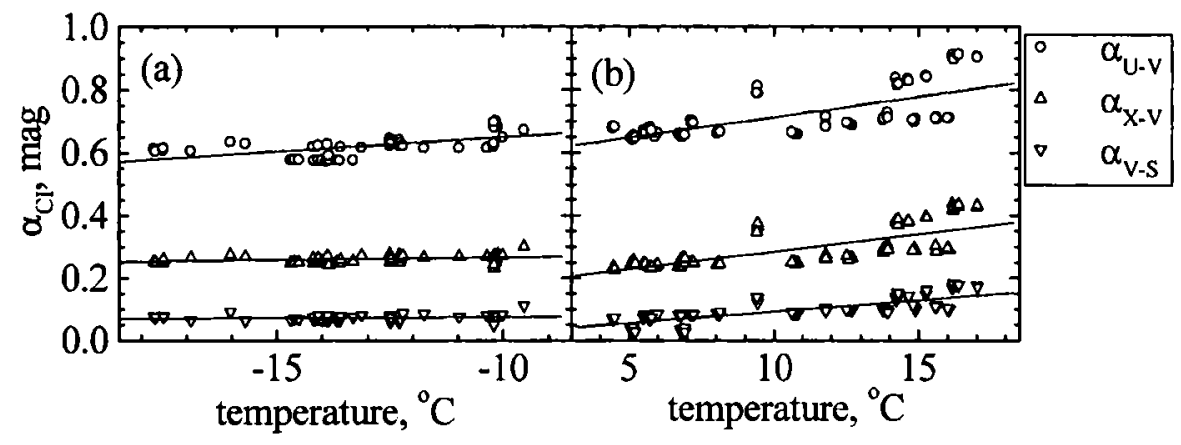

Fig. 3. Temperature dependence of extinction coefficients within two intervals of absolute humidity: (a) between 1 and $2 \mathrm{~g} / \mathrm{m}^{3}$ and (b) between 6 and $7 \mathrm{~g} / \mathrm{m}^{3}$. 
midity depends on the nature and chemical composition of primary aerosols (Zujev \& Krekov 1986).

Theoretical calculations show that the aerosol extinction grows with relative humidity. This is related to changes in aerosol size distribution and complex refraction index: when the relative humidity grows, the process of condensation intensifies, the number of large particles increases and refraction index decreases. But these calculations are valid only for a stable atmosphere. When meteorological conditions vary, the dependence of extinction coefficient on relative humidity can go into reverse (Zujev \& Krekov 1986).

With observational material available for Molètai Observatory it has been possible to construct plots showing the dependence of extinction on both absolute and relative humidity (Figs. $2 \mathrm{c}$ and $2 \mathrm{~d}$ ). As is apparent from these plots, the extinction coefficients are nearly free of relative humidity dependence, but they follow a clear growth with absolute humidity. The latter trend can be explained by the temperature dependence of extinction.

Although the dependence of the extinction on temperature is evident, its relation to absolute humidity, judging from our data, is rather uncertain. Binning our data in narrow temperature intervals $\left(5^{\circ} \mathrm{C}\right)$ appeared to be of no help: in one of the temperature intervals the extinction increases with absolute humidity, while in the other intervals it decreases with increasing humidity.

To check whether the theoretical dependence of extinction on relative humidity is valid for Moletai, our data were investigated for every month separately, taking into account the seasonal aerosol variations. The growth of the extinction with relative humidity was detected just in February, May and August. In other months, the extinction was either independent of the relative humidity or even decreasing with increasing air-moisture. Due to the fact that, during the night, relative humidity is growing when the temperature falls and that some aerosols are settling down on to the ground, we can, in such a case, observe the transparency of the atmosphere getting better throughout the night.

Thus we can conclude that the extinction variations are more related to temperature variations than to humidity. Rather, the humidity dependence is negligible. 


\subsection{Dependence on pressure}

The above analysis of the dependence of the extinction coeffcients on temperature and humidity was made under the assumption that the effect of atmospheric pressure on extinction was negligible. Special investigations, based on values of the extinction coefficients determined within narrow temperature $\left(5^{\circ} \mathrm{C}\right)$ and humidity $\left(1 \mathrm{~g} / \mathrm{m}^{3}\right)$ intervals, have really shown no reliable correlation between the extinction and pressure.

3.4. Possible explanation of the growth of extinction with temperature

On the basis of the above conclusion that pressure and humidity practically have no influence on the atmospheric extinction, and, also, allowing for a poor temperature dependence of Rayleigh scattering (Pakštiené et al. 2001), we may affirm that the number and size distribution of aerosol particles are mainly responsible for the temperature dependence of extinction coefficients. This may be connected, in one or another way, with the seasonal changes in the atmosphere. During a warm season, the atmosphere can be contaminated by a lot of pollen and urban dust. The smoke also rises high and keeps in the atmosphere for a longer time. Besides that, a large amount of particles can serve as additional condensation nuclei, increasing the number of water droplets in the atmosphere. As direct measurements by atmosphere physicists show, the number of aerosol particles in the lower atmosphere is maximal in winter time. Because of small atmospheric convection during this period of a year, aerosol remains in the lower layers of the atmosphere and has a little influence on astronomical observations.

\section{THE DEPENDENCE OF EXTINCTION ON THE AEROSOL SIZE DISTRIBUTION}

\subsection{Method of calculation of the aerosol size distribution}

To calculate the size distribution of aerosol particles at a given moment of observation, it is necessary to know: (1) a model of particle size distribution, (2) formulas for Mie scattering, (3) a model for extinction in the Earth atmosphere, (4) the extinction coefficients for differing passbands (the more passbands used, the better results that can be achieved). 
(1) The distribution of the aerosol particles with sizes in the interval from 0.06 to $10 \mu \mathrm{m}$ may be well described by the Junge size distribution (McCartney 1977) in the form

$$
N(r)=u r^{-v}
$$

where $N(r)$ is the concentration of particles with radius $r$, in $\mathrm{cm}^{-3}$, $u$ is the Junge multiplier and $v$ is the Junge exponent.

For the particle size interval mentioned above a logarithmic plot of the size distribution gives a straight line the slope of which is defined by the Junge exponent. The Junge multiplier depends on concentration of particles. For a haze, the exponent takes values from 4 to 5 , whereas the Junge multiplier can be very small (about (2$3) \cdot 10^{-4}$ ). Meanwhile, in the case of fog the exponent value is about 3 , but the multiplier can be some hundreds (the larger the radius at the maximum of function $N(r)$, the larger the Junge multiplier (McCartney 1977).

(2) On the basis of Mie theory, the extinction coefficients can be calculated by the formula (Trakhovsky \& Shettle 1987)

$$
\alpha_{\mathrm{m}}(\lambda)=\int K_{\mathrm{m}}(r, \lambda) \frac{\delta v}{\delta \log r} d \log r
$$

or

$$
\begin{gathered}
\alpha_{\mathrm{m}}(\lambda)=\sum_{r=r_{1}}^{R} K_{\mathrm{m}}(r, \lambda) \Delta v \\
\Delta v=4 \pi r^{2} \Delta r \Delta N
\end{gathered}
$$

where $\lambda$ is the wavelength, $\Delta v$ is the volume interval (the length of particle size interval), $\Delta N$ is the number of particles in the volume interval $\Delta v, m=m_{\mathrm{r}}-i m_{\mathbf{i}}$ is the complex refractive index with its real and imaginary parts $m_{\mathrm{r}}$ and $m_{\mathrm{i}}$, and $K_{\mathrm{m}}$ denotes the Mie extinction kernel.

As was already mentioned, the nature of aerosols at Molettai and Maidanak is different, so the complex refraction indices for both observatories are different too. (The imaginary part of the refraction index will not be discussed here, as in the calculations only the real part is used, which, for convenience, will be called the refraction index.) 
The refraction index of aerosols depends on the wavelength of scattered light and on the nature of aerosols (e.g., when the wavelength is $500 \mathrm{~nm}$, the real part of the refraction index is 1.48 for silicates, 1.54 for dust of soil, 1.50 for volcanic ashes, 1.82 for soot, 1.45 for organic matter, and 1.33 for water). Because atmospheric aerosol consists of particles of a different nature, its optical characteristics are described by some average value of the refraction index. When the composition of aerosol changes, the average of refraction index changes too, e.g., when relative humidity increases, refraction indexes decrease and extinction grows. Average values of the refraction index for many sites and for different humidity can be found in the literature (see, e.g., Zujev \& Krekov 1986). If we accept, that, at Moletai, aerosols are of urban origin and that observations are mainly carried out when relative humidity is between 85 and 90 percent, the refraction index to be used in the optical domain is 1.4 (Zujev \& Krekov 1986). For Maidanak, where aerosols are made mostly from silicate and dust from the ground (Zujev \& Krekov 1986), a refraction index of 1.5 was chosen.

As a first approximation to the extinction kernel one might choose the anomalous diffraction kernel developed by van de Hulst (1957):

$$
K_{\mathrm{m}}(r, \lambda)=\frac{3 c k}{4}\left[\frac{2}{\rho}-\frac{4 \sin \rho}{\rho^{2}}+\frac{4(1-\cos \rho)}{\rho^{3}}\right],
$$

where $c=0.001$ is a unit-dependent constant, with $K_{\mathrm{m}}$ in $\mathrm{km}^{-1} \mu \mathrm{m}^{-3}$ $\mathrm{cm}^{3}$ and $k$ in $\mu \mathrm{m}^{-1}$,

$$
\begin{gathered}
\rho=4 \pi r \frac{m_{r}-1}{\lambda}, \\
k=\frac{2 \pi}{\lambda} .
\end{gathered}
$$

(3) Rayleigh scattering and ozone absorption are calculated accepting the same model for extinction in the Earth atmosphere as in Paper II.

(4) The aerosol extinction at Molètai and Maidanak was taken as the difference between the observed extinction coefficients and the calculated Rayleigh plus ozone extinction.

The optimal Junge distribution was found by iteratively calculating the extinction coefficients (Eq. 3). The whole procedure was 
repeated with the Junge coefficients changed by small steps until the least squares fit to the observed values of extinction coefficients was obtained.

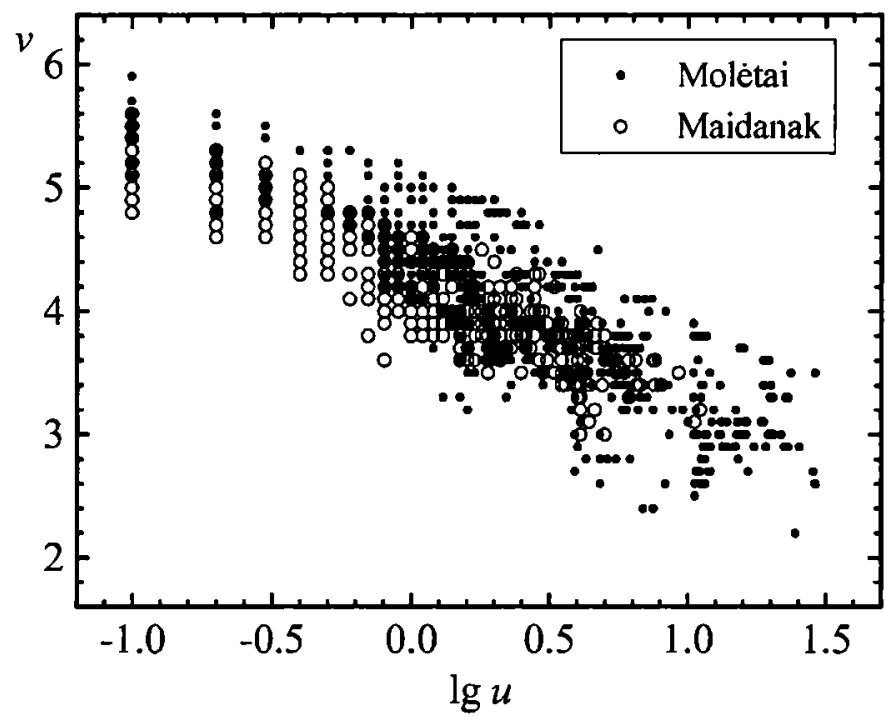

Fig. 4. Interrelation between the Junge coefficients $\log u$ and $v$ for Moletai and Maidanak observatories.

\subsection{Junge coefficients}

The Junge coefficients $u$ and $v$, calculated using the above procedure for Moletai, vary within a wider interval than those calculated for Maidanak, but for both sites the Junge exponent is less than 6. As can be seen from $v, \log u$ diagram (Fig. 4.), the points are spread over some area. This dependence cannot be approximated by a straight line, because the same value of the exponent $v$ can correspond to different numbers of particles and hence value of log $u$. For Moletai, this area is wider and lies at higher values of $v$ than for Maidanak, especially when the Junge multiplier $u$ is large. This means that for the same Junge exponent $v$, the number of aerosol particles at Moletai varies within a wider interval and often is greater than at Maidanak.

At Maidanak, during most of the nights the Junge multiplier $u$ is close to zero, while for Molètai its typical values are close to 
Table 1. The differences between the observed and calculated (Eqs. 3 and 6) extinction coefficients.

\begin{tabular}{lllllllr}
\hline & $\Delta \alpha_{\mathrm{U}}$ & $\Delta \alpha_{\mathrm{P}}$ & $\Delta \alpha_{\mathrm{X}}$ & $\Delta \alpha_{\mathrm{Y}}$ & $\Delta \alpha_{\mathrm{Z}}$ & $\Delta \alpha_{\mathrm{V}}$ & $\Delta \alpha_{\mathrm{S}}$ \\
\hline Molètai & 0.020 & -0.016 & -0.005 & -0.005 & 0 & 0 & 0.005 \\
Maidanak & 0 & -0.004 & 0 & 0.005 & 0 & 0.005 & -0.005 \\
\hline
\end{tabular}

2. The maximum value of $u$ are 30 for Molètai and only 12 for Maidanak. The Junge exponent $v$ is also different for both sites: the most frequent values are close to 4.5 and 4 for Molètai and Maidanak, respectively.

\subsection{Accuracy of the method}

To evaluate how good are the extinction values calculated by Mie theory with the determined parameters of the Junge distribution, the differences between the observed and calculated (Eqs. 3 and 6) extinction coefficients have been examined. The typical values of these differences are given in Table 1 . The largest discrepancies $(0.020$ and $0.016 \mathrm{mag}$ ) are found for the Moletai extinction coefficients in $U$ and $P$. For Maidanak, the differences for all passbands are less than, or equal to, 0.005 mag. A reasonable explanation for this seems to be that the aerosol size distribution is linear on a logarithmic scale (Junge distribution) at Maidanak and non-linear at Molètai.

To get the size distribution in a more explicit form, it has to be divided into smaller intervals of particle sizes, within which real particle distributions should be determined iteratively by varying the values of aerosol concentration (Heintzenberg et al. 1981, Jiménez et al. 1998).

\subsection{Concentration of aerosols}

Despite the generally rough form of the Junge size distribution, its use can be exemplified by investigating the dependence of the extinction coefficients on parameters of the Junge distribution and on concentration of the aerosol particles.

The histograms of the aerosol concentrations calculated for Moletai and Maidanak are shown in Figs. 5a and 5b, respectively. At Moletai, the concentration is mostly around 2000 particles $/ \mathrm{cm}^{3}$. 


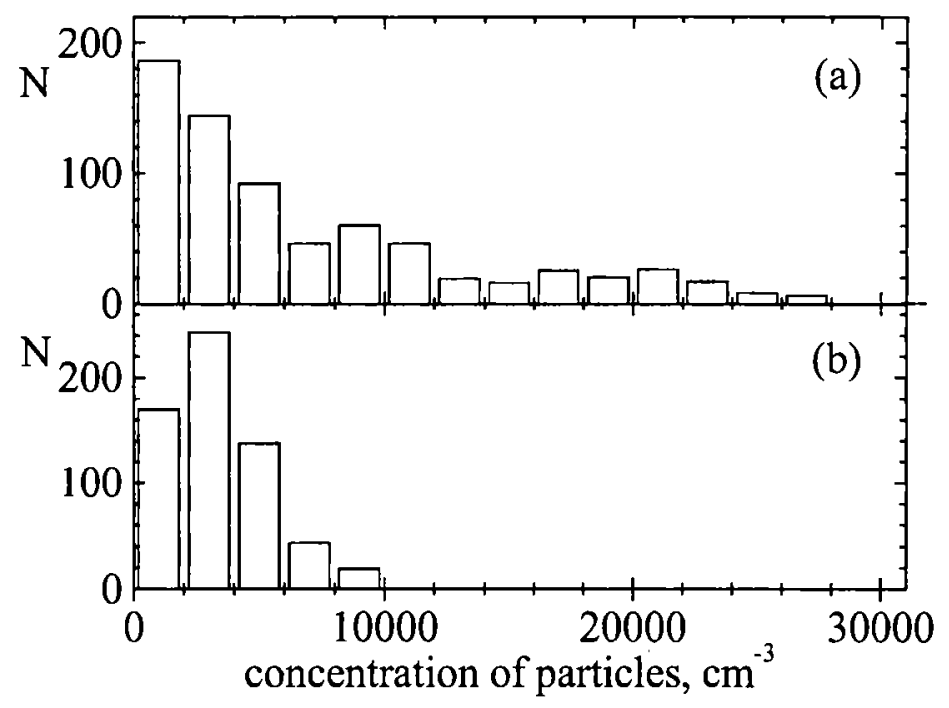

Fig. 5. The histogram of the calculated aerosol concentrations for (a) Molètai and (b) Maidanak.

At Maidanak, it is higher and varies, in most cases, between 2000 and 4000 particles $/ \mathrm{cm}^{3}$. Here, the aerosol concentration drops faster and reaches the maximum value of only about 10000 . At the same time, the decrease in aerosol concentration at Moletai is slow, but the maximum value reached is about 27000 particles $/ \mathrm{cm}^{3}$. High aerosol concentration is usually observed in warm seasons when the extinction coefficients for color indices are also largest (Paper II). Although the concentration of aerosols at Maidanak is more stable, a significant number of nights with negligible aerosol component is found at Molètai.

To see what effect the refraction index has on aerosol size distribution, the procedure described in section 4.1 was repeated for the Moletai data, but with a refraction index of 1.5 (as for Maidanak) instead of 1.4. The extinction coefficients, calculated with a larger refraction index, are smaller, and hence the concentration of aerosol particles increases. In this case, the typical concentration of aerosols at Moletai is around 3000 particles $/ \mathrm{cm}^{3}$, and the maximum concentration is as high as 29000 particles $/ \mathrm{cm}^{3}$.

The present results are in good agreement with those, obtained by other authors for other places. According to McCartney (1977), the typical aerosol distribution at Minneapolis (by 1967 data) has Junge coefficients $u=2.49$ and $v=4$, which correspond to a con- 
centration $N=4000$ particles $/ \mathrm{cm}^{3}$. In the same work, the particle size distribution of continental aerosol is given with the same Junge exponent $v=4$, but the Junge multiplier $u$ can vary in a wide interval. In Seattle, the concentration of particles varies from 3000 to 45000 particles $/ \mathrm{cm}^{3}$ (Pueschel \& Noll 1967). The dependence of extinction on aerosol size distribution was investigated by Jiménez et al. (1998), who used the size distributions measured at Timbuctu and Agadez for highly transparent and dusty (with dust clouds from the Sahara desert) periods, when the concentration of aerosols was around 2000 and 12000 particles $/ \mathrm{cm}^{3}$, respectively.

It should be noted that small particles constitute a major part of aerosols, which are distributed mostly close to the surface of earth (in the troposphere). With higher altitude, the number of small particles decreases, whereas larger particles increase in number, but small particles always remain more numerous (McCartney 1977). Because of high altitude the concentration of aerosols above Maidanak is , in many cases, lower than above Molètai.

\subsection{Changes in extinction and aerosol size distribution at Moletai during the night of April 16, 1993}

Knowledge of the extinction coefficients, Junge parameters and concentration makes it possible to judge the processes in the Earth's atmosphere. As an example, we consider the night of April 16, 1993, which had the largest extinction coefficients observed throughout all nights used in this investigation. The instantaneous extinction coefficients as a time function are plotted for the magnitude $V$ (Fig. 6a) and for three color indices of the Vilnius system (Fig. 6d). As we can see, all of the extinction coefficients for color indices gradually decrease. A sudden increase in extinction for only the magnitude indicates, that it is due to the neutral (grey) extinction. The behavior of the Junge parameters during the night is shown in Figs. $6 \mathrm{~b}$ and $6 \mathrm{e}$, together with the concentration variations of aerosols shown in Fig. 6c. The comparison of these plots leads to the following interpretation. From the beginning of the night, the extinction in magnitude $V$ and in all color indices as well as the concentration and, maybe, the Junge exponent are slightly diminishing. This is, undoubtedly, due to the slow increase in aerosol sizes (slow condensation). At midnight, a sudden increase in extinction for all magnitudes, but not any in extinction for color indices, indicates the change in aerosol size distribution due to an increase in the number of large parti- 


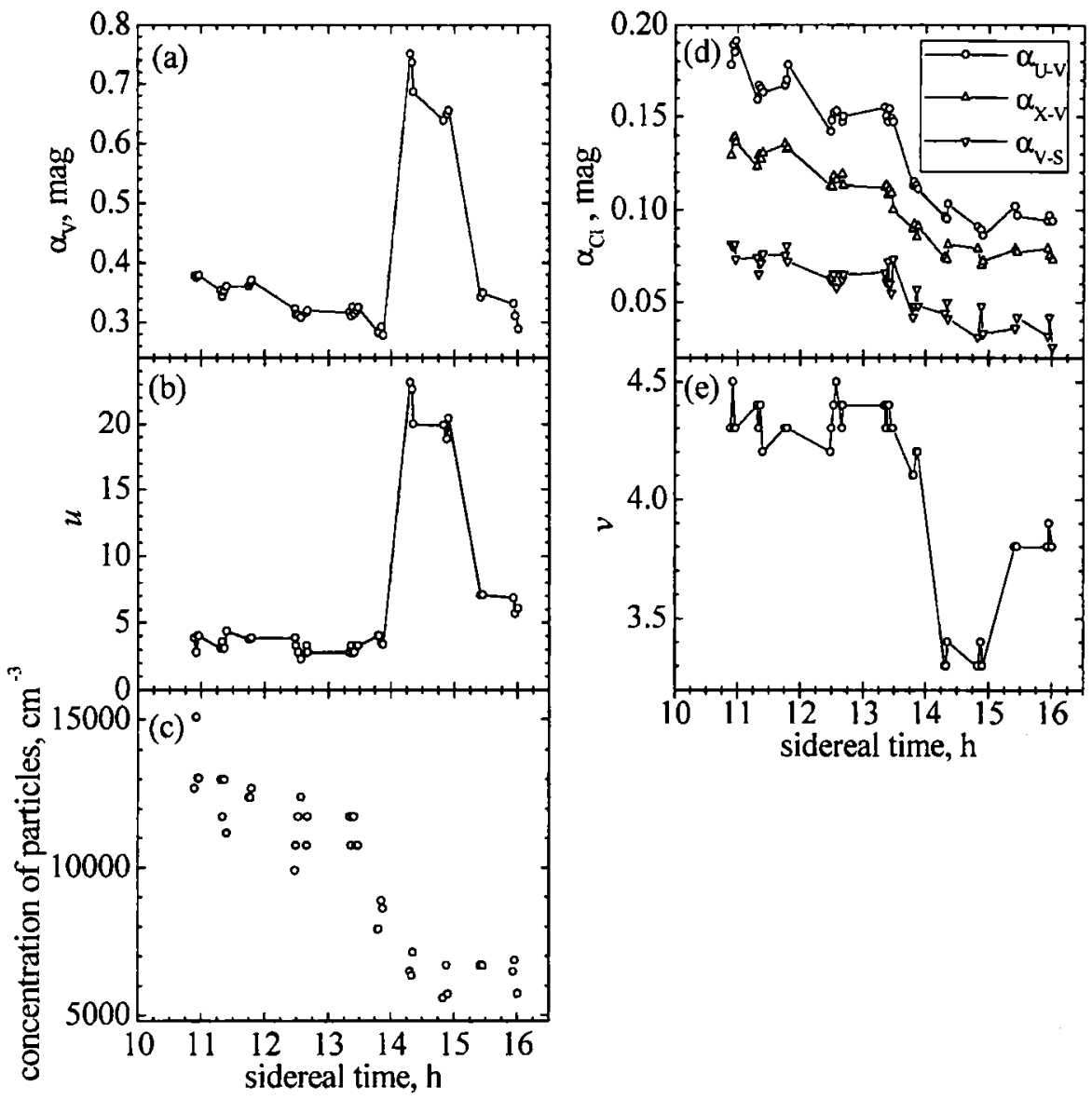

Fig. 6. Panels (a) and (d): the instantaneous extinction coefficients for the magnitude $V$ and three color indices of the Vilnius system. Panels (b) and (e): Junge coefficients for the Junge multiplier and exponent, respectively). Panel (c): the concentration of particles as function of time at the Moletai Observatory during the night of April 16, 1993.

cles. A sudden drop-off to almost half of the aerosol concentration shows that the process of condensation might have taken place. After that, the concentration remained stable until the morning, and the extinction coefficients for color indices also showed no appreciable variations. The decrease of extinction for only magnitudes and increase of the Junge exponent indicate a diminution in the number of large particles. 
4.6. Correlation between the Junge coefficients and the parameters of aerosol extinction

The Junge distribution (Eq. 1) and the aerosol extinction $\left(\alpha_{\text {aer }}=\right.$ $A_{0} \lambda^{-b}$ ) (see Paper II) are defined by the equations of closely similar form. Thus, the correlation between the coefficients of both equations should hold. To find this correlation, the aerosol parameters $A_{0}$ and $b$ were calculated for both Moletai and Maidanak observatories with the help of synthetic extinction coefficients (Eq. 3). By means of the $b, v$ diagram, we find that the dependence of $v$ on $b$ is non-linear, but appears to be the same for both observatories even if $u$ and $A_{0}$ are different (Fig. 7a). This dependence can be written in the following polynomial form, which is valid for the $b$ interval from 0 to 1.7:

$$
\begin{aligned}
v= & 2.09+10.51 b-47.46 b^{2}+126.27 b^{3}-183.06 b^{4}+ \\
& 146.03 b^{5}-60.08 b^{6}+9.97 b^{7}
\end{aligned}
$$

The empirical relationship between the Junge exponent $v$ and the aerosol extinction exponent $b$ has been described also by Forbes et al. (1996). Here, the parameters $v$ and $b$ are expressed through the same variable $\gamma$ :

$$
\begin{aligned}
& v=\gamma+1, \\
& b=\gamma-2 .
\end{aligned}
$$

Then, from Eqs. 10 and 11 we get a linear dependence:

$$
v=b+3 \text {. }
$$

Junge exponent $v$, calculated by Eq. 12 , is smaller, when $b \leq 0.3$, and greater, when $b \geq 1.0$, than the values calculated in this work.

The relationship between $u$ and $v$ appears to be linear, but the slope itself depends on the parameter $b$ of aerosol extinction (or on the Junge exponent $v$ ) (Fig. 8):

$$
u\left(A_{0}, b\right)=d(b) A_{0}
$$

The $u, A_{0}$ diagrams for Moletai and Maidanak are shown in Figs. $8 \mathrm{a}$ and $8 \mathrm{~b}$, respectively, where the dots denote the observed extinctions, and the lines show the relations between $u$ and $A_{0}$ for constant $b$ values. The slopes $d$ of the lines $u, A_{0}$ were found for 

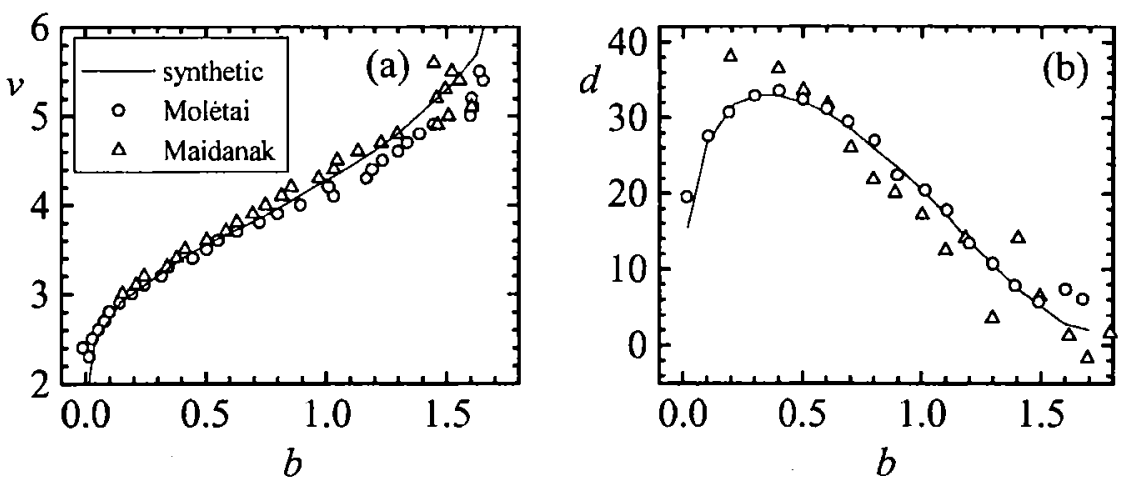

Fig. 7. Dependence of the Junge exponent $v$ (panel a) and the slope $d$ of the $u\left(A_{0}\right)$ line (panel b) on the aerosol extinction exponent $b$. Solid line - dependences determined from synthetic extinction coefficients and fitted by polynoms, triangles and circles - results from observed extinction coefficients.

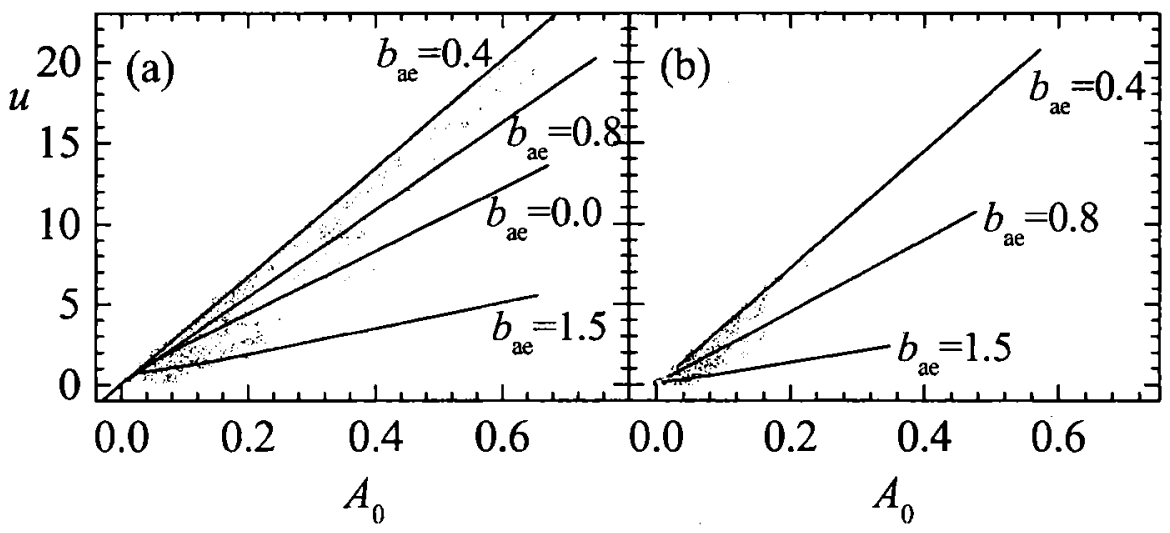

Fig. 8. Dependence of the Junge multiplier $u$ on the aerosol extinction parameter $A_{0}$ for Molètai (a) and Maidanak (b).

different values of $b$ (with a step of 0.1 ) using synthetic extinction coefficients, calculated by Eq. 3. The dependence of $d$ on $b$, estimated from the synthetic extinction coefficients (solid line) is shown in Fig. 7b. This dependence can be written as the sixth order polynomial valid for the interval of $b$ values from 0 to 1.7 :

$$
\begin{aligned}
d(b)= & 12.88+170.78 b-515.39 b^{2}+738.02 b^{3}- \\
& 579.65 b^{4}+229.20 b^{5}-35.25 b^{6} .
\end{aligned}
$$


In Fig. 7, the dependences of the Junge coefficients on the parameters $A_{0}$ and $b$ of aerosol extinction, $u\left(A_{0}\right)$ and $v(b)$, calculated from synthetic extinction coefficients are compared with the results obtained from observed extinction coefficients. The line shows the synthetic results, and the circles and triangles represent the results obtained from extinction observations at Moletai and Maidanak, respectively. The points plotted are means in the intervals $\Delta v=0.1$ (Fig. 7a) and $\Delta b=0.1$ (Fig. 7b). As is seen from the figures, the results obtained using synthetic and observed extinction coefficients are very similar. The observed $v(b)$ points for Moletai lie slightly below the synthetic dependence. It can be caused by a non-linear (other than Junge) aerosol size distribution at Moletai, what gives larger errors in estimated parameters $v$ and $b$. As regards $u\left(A_{0}\right)$, the synthetic and the observed slopes $d(b)$ for Moletai are practically the same, except for two points at $b \geq 1.6$. These points come from small number of observations, therefore their reliability is low, too. For Maidanak, the synthetic and observed slopes $d(b)$ are slightly different, because the parameters $u$ and $A_{0}$ vary in much narrower interval than for Moletai and hence the slopes of $u\left(A_{0}\right)$ are estimated with a larger error.

Using Eqs. 9, 10 and 11 we can calculate a rough Junge distribution for every moment of observations, if only we know the aerosol extinction parameters $b$ and $A_{0}$.

\section{SUMMARY AND CONCLUSIONS}

Short-term variations of atmospheric extinction cannot be conditioned by variable meteorological parameters alone. Atmospheric extinction depends on seasonal changes in composition and concentration of aerosols and also on short-term atmospheric phenomenon such like rain or drought. For instance, just after rain the amount of solid aerosol particles (dust) is smaller and of watery particles (vapor) is greater than during drought.

We have found that at Moletai the atmospheric extinction slightly increases with increasing temperature. At the same time, absolute humidity increases too. Therefore, it is difficult to separate both effects. The increase of extinction with increasing temperature may be explained by both the seasonal increase of the number of the aerosol particles and change of its size distribution. 
The dependence of extinction on relative humidity, which is well known from other studies (Zujev \& Krekov 1986), was not found from our data, possibly because of unstable atmosphere at Moletai. The extinction was also found to be practically independent on pressure.

For Maidanak, for most of the nights the Junge multiplier is close to zero, and, for Moletai, its most frequent value is close to 2 . The maximal value of the Junge multiplier reaches 30 for Moletai, and only 12 for Maidanak. The Junge exponent is also greater for Moletai. Its most frequent values are close to 4.5 and 4 for Molètai and Maidanak, respectively.

The most frequent concentration of the aerosol particles found is 4000 to 6000 particles $/ \mathrm{cm}^{3}$ for Molètai and 2000 to 4000 particles $/ \mathrm{cm}^{3}$ for Maidanak. The maximal observed concentration is about 28000 particles $/ \mathrm{cm}^{3}$ for Moletai and 10000 particles $/ \mathrm{cm}^{3}$ for Maidanak. In both sites, a few nights with very small concentration of aerosol were observed.

The Junge size distribution coefficients $u$ and $v$, and the aerosol extinction parameters $A_{0}$ and $b$ are interdependent. The dependence $u\left(A_{0}\right)$ is linear with slope $d(b)$. The dependences $v(b)$ and $d(b)$ are non-linear, but can be written as linear equations for $b$ values between 0.2 and 1.5 in case of $v(b)$, and between 0.5 and 1.6 in case of $d(b)$. When we have the dependences $v(b)$ and $u\left(A_{0}, b\right)$, we can estimate a rough Junge size distribution for every moment of extinction observation.

ACKNOWLEDGMENTS. The author is thankful to S. Bartašiūtè, A. Kazlauskas, R. Mikutavičienė and J. Sperauskas for their observations of extinction stars, and to V. Straižys, J. Sūdžius and $\mathrm{K}$. Zdanavičius for helpful advice, comments and discussion, and to the Utena Meteorological Station, which let use the data of meteorological conditions. The support provided by the Lithuanian State Science and Studies Foundation (1999 and 2000 Ph.D. student program) is also acknowledged.

\section{REFERENCES}

Dave J. V. 1969, Applied Optics, 8, 155

de Rooij W. A., van der Stap C. C. A.H. 1984, A\&A, 131, 237

Forbes M. C., Dodd R. J., Sullivan D. J. 1996, Baltic Astronomy, 5, 281

Gutiérrez-Moreno A., Moreno H., Cortes G. 1982, PASP, 94, 722

Hayes D. S., Latham D. W. 1975, ApJ, 197, 593 
Heintzenberg J., Müller H., Quenzel H., Thomalla E. 1981, Applied Optics, 20,1308

Jiménez A., Gonzalez Jorge H., Rabello-Soares M. C. 1998, A\&AS, 129, 413

Lockwood G. W., Thompson D. T. 1986, AJ, 92, 976

McCartney E. J. 1977, Optics of the Atmosphere, John Wiley \& Sons, London

Pakštienè E., Zdanavičius K., Bartašiūtè S. 2001, Baltic Astronomy, 10, 439 (Paper II)

Pueschel R.F., Noll K. E. 1967, J. Appl. Meteorol., 6, 1045

Reimann H. G., Ossenkopf V., Beyersdorfer S. 1992, A\&A, 265, 360

Shah G. A. 1985, Astron. Nachr., 306, 63

Trakhovsky E., Shettle E. P. 1987, Applied Optics, 26, 5148

van de Hulst H. C. 1957, Light Scattering by Small Particles, Wiley, N.Y. Wiscombe W. J. 1980, Applied Optics, 19, 1505

Zujev V.E., Komarov V.S. 1986, Statistics Models of Temperature and Gas Component of the Atmosphere, Hidrometeoizdat Publ. House, Leningrad (in Russian)

Zujev V.E., Krekov G. M. 1986, Optical Models of the Atmosphere, Hidrometeoizdat Publ. House, Leningrad (in Russian)

Yamamoto G., Tanaka M. 1969, Applied Optics, 8, 447 
